\title{
An SCF-stabilization approach to excited states embedded in the continuum ${ }^{a)}$
}

\author{
C. F. Bender \\ Theoretical Atomic and Molecular Physics Group, Lawrence Livermore Laboratory, University of \\ California, Livermore, California 94550
}

Vincent McKoy

Arthur Amos Noyes Laboratory of Chemical Physics, ${ }^{\text {b) }}$ California Institute of Technology, Pasadena, California 91125

Ernest R. Davidson

Department of Chemistry, University of Washington, Seattle, Washington, D. C. 98195

By using SCF and stabilization-like procedures, we have located a $\left(\pi, \pi^{*}\right)$ singlet resonance-like state in ethylene at $10.21 \mathrm{eV}$. This state is embedded in the ionization continuum and carries an oscillator strength of 0.46 and is probably the analog to the spectroscopic $V$ state in Hartree-Fock theory. Implications of these results for other systems are discussed.

\section{INTRODUCTION}

Recently there have been many calculations designed to characterize the $\left(\pi, \pi^{*}\right)$ singlet state of ethylene. ${ }^{1,2}$ Much of this interest stems from the SCF calculations of Dunning et al. ${ }^{3}$ which showed that the inclusion of diffuse orbitals along $w$ ith valence types gave $a^{1}\left(\pi, \pi^{*}\right)$ state which was essentially Rydberg-like, e.g., with a second moment of the $\pi^{*}$ orbital, $\left\langle\pi^{*}\left|x^{2}\right| \pi^{*}\right\rangle$, of $42 a_{0}^{2}$ where $x$ is perpendicular to the plane of the molecule. This result is surprising and does seem inconsistent with experimental evidence such as the absence of any significant shift in this transition when the spectrum is observed in solid or liquid krypton. ${ }^{4}$ Several conventional calculations designed to include the effects of electron correlation on the location and nature of this state also concluded that the $V$ state of ethylene had considerable diffuse or Rydberg character. ${ }^{1,2}$ Recently, Davidson, ${ }^{5}$ using traditional large basis set configuration interaction methods, has shown that the $V$ state of ethylene is in fact relatively valence-like. These results along with an adequate review of the background and other relevant aspects of this problem are discussed in Ref. 5. Their conclusions agree with the earlier work of Shibuya and McKoy, ${ }^{6}$ Tanaka, ${ }^{7}$ and Iwata and Freed. ${ }^{8}$

The purpose of the present work is not to obtain a description of the true ${ }^{1}\left(\pi, \pi^{*}\right)$ state of ethylene but rather to try to understand the nature of the Hartree-Fock $\left(\pi, \pi^{*}\right)$ spectrum in terms of a simple physical model. Such a model can display the essential physics of the problem and can be used to interpret and predict the existence of similar effects in the Hartree-Fock description of other molecules, e.g., $\mathrm{N}_{2}$ and $\mathrm{CO}_{2}$. Our model of the Hartree-Fock spectrum of the ${ }^{1}\left(\pi, n \pi^{*}\right)$ states in ethylene is based on the observation that, in the SCF approximation, the lowest state of the same symmetry as the spectroscopic $V$ state $\left({ }^{1} B_{1 u}\right)$ is in fact the first member of a Rydberg series followed by the other members

\footnotetext{
a) This work was performed under the auspices of the U. S. Energy Research and Development Administration under contract No. W-7405-Eng-48.

${ }^{\text {b) }}$ Contribution No. 5455 .
}

of this series leading up to the ion at $8.96 \mathrm{eV}$. Hence SCF calculations with basis sets successively augmented with diffuse functions should yield additional members of this Rydberg series. The present calculations actually give up to five members of the series with energies which can be fitted to the Rydberg formula and with oscillator strengths ranging down from the largest value of 0.09 . None of these states shows the expected characteristics of an intravalence transition such as the $N$ $-V$ transition. The question that now arises is what and where is the analog to the spectroscopic $V$ state in Hartree-Fock theory. We have previously suggested that what would be the $V$ state in Hartree-Fock theory has been pushed into the Hartree-Fock ionization continuum and is heavily contaminated with the lower-energy Rydberg states due to orthogonality requirements. ${ }^{9}$ In this paper, we carry out a series of SCF calculations on the sing let $\left(\pi, n \pi^{*}\right)$ states of ethylene which locates a state embedded in the continuum at $10.21 \mathrm{eV}$ which is very probably the SCF analog of the spectroscopic $V$ state. Moreover, the location and nature of this state is stable with respect to major changes in the composition of the basis set.

In the next section, we will discuss the SCF and stabilization procedure which we use to carry out these calculations and then present the results for the $\left(\pi, n \pi^{*}\right)$ states in ethylene. Finally, we outline the implications of these results for electronic transitions in other systems, e.g., $\mathrm{N}_{2}, \mathrm{CO}$, and $\mathrm{CO}_{2}$ and show that these states embedded in the continuum with large oscillator strengths can lead to definite problems in using such discrete spectral distributions to derive photoionization cross sections.

\section{THEORETICAL ASPECTS AND RESULTS}

The basis of our model of the $\left(\pi, n \pi^{*}\right)$ or $1 b_{34}(\pi)$ $\rightarrow n b_{2 g}\left(n \pi^{*}\right)$ spectrum of ethylene in the SCF approximation is that those states with this electron configuration which are below the first ionization potential are all members of a Rydberg series. This model seems to imply that the analog of the valence-like $\left(\pi, \pi^{*}\right)$ state in 
TABLE I. Summary of SCF calculations locating a $\left(\pi, \pi^{*}\right)$ resonance-like state. ${ }^{a, b}$

\begin{tabular}{|c|c|c|c|c|c|c|}
\hline$N$ & $R_{1}$ & $R_{2}$ & $R_{3}$ & $R_{4}$ & $R_{5}$ & $R_{5}^{*}$ \\
\hline 8.91 & 7.43 & 7.35 & 7.35 & 7.35 & 7.35 & 7.33 \\
\hline \multirow[t]{6}{*}{19.84} & 9.93 & 8.09 & 8.06 & 8.06 & 8.06 & 8.04 \\
\hline & $2 \overline{0.95}$ & 10.17 & 8.38 & 8.38 & 8.38 & 8.36 \\
\hline & & $\overline{21.11}$ & 10.21 & 8.60 & 8.60 & 8.58 \\
\hline & & & 21.13 & 10.21 & 8.77 & 8.75 \\
\hline & & & & $\overline{8}$ & 10.21 & 10.21 \\
\hline & & & & & 21.14 & 19.81 \\
\hline
\end{tabular}

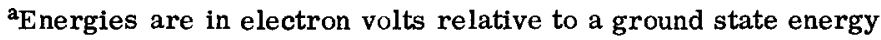
of -78.04171 a. u. See text for a discussion of the basis sets $N, R_{1}, \cdots, R_{5}, R_{5}^{*}$. We list the energies of all the states except the one at 2.55 a. u. above the ground state.

b The arrows follow the state which we identify as a $\left(\pi, \pi^{*}\right)$ resonance. The SCF ionization potential in these calculations is $0.96 \mathrm{eV}$.

SCF theory has been pushed beyond the ionization potential and hence should appear as a resonance embedded in the continuum. What would some characteristics of such a state be? We can expect that it would be heavily contaminated with the lower-energy Rydberg states (because of orthogonality) and hence would have more diffuse character, e.g., a larger second moment, than the true spectroscopic $V$ state. On the other hand the SCF $V$ state should have a large transition moment and oscillator strength for the ${ }^{1} A_{g} \rightarrow B_{1 u}$ absorption due to its valence character.

To locate this SCF state embedded in the continuum, we carry out a series of SCF calculations on the $\left(1 b_{3_{u}}\right.$, $n b_{2 g}$ ) states and look for a state in the continuum which is stable with respect to extensive changes in the basis set. As the number of diffuse orbitals in the basis is increased, we should obtain additional members of the Rydberg series and observe very little effect on the location and character of the resonance-like state or on the previously converged Rydberg states. This approach to locating such a state in the continuum is essentially a stabilization method which has been widely used in describing resonant scattering. ${ }^{10}$ The necessary spin-restricted excited state SCF calculations were carried out on these states following the excited state method der scribed by Stenkamp and Davidson. ${ }^{11}$ This method allows excited states having the same orbital symmetry as lower states to be obtained by a variationally stable procedure.

For these calculations, we used six different basis sets and obtained all the $\left(\pi, n \pi^{*}\right)$ states given by each basis set. These basis sets are referred to as $N, R_{1}$, $R_{2}, R_{3}, \ldots, R_{5}$ where the basis set $N$ is a $[3 s 2 p 1 d / 2 s]$ valence-like Gaussian orbital basis with Dunning's $s$ and $p$ contractions ${ }^{12}$ and a $d$-orbital exponent of 0.75 . All six $d$ functions were included. The basis sets $R_{1}$ through $R_{5}$ contain the basis set $N$ successively augmented by $p_{x}$ basis functions on the carbon atoms (the molecule is in the $y z$ plane) with exponents of $0.0251,0.0063,0.0016$, 0.0004 , and 0.0001. For example, the basis sets $R_{1}$ and $R_{5}$ contain three and seven $p_{x}$-type functions, respectively.
The energies of the $\left(\pi, n \pi^{*}\right)$ states obtained from these size calculations are listed in the corresponding columns of Table I. A clear trend emerges from these calculations. In the $N$ basis the only relevant state is the one at $8.91 \mathrm{eV}$. Adding a single diffuse function on each $\mathrm{C}$ atom to the $N$ basis gives a state at $7.43 \mathrm{eV}$ and another at $9.93 \mathrm{eV}$ which is hence in the continuum (HartreeFock $I P=8.96 \mathrm{eV}$ ). In going to the $R_{2}$ basis the state at $7.43 \mathrm{eV}$ in the $R_{1}$ calculation moves slightly down to $7.35 \mathrm{eV}$, another (Rydberg) state appears at $8.09 \mathrm{eV}$, and the state at $9.93 \mathrm{eV}$ is pushed up to $10.17 \mathrm{eV}$. This trend continues on through the calculations with basis sets $R_{3}$ and $R_{4}$ until we finally have five Rydberg states at energies $7.35,8.06,8.38,8.60$, and $8.77 \mathrm{eV}$ and a stabilized root at $10.21 \mathrm{eV}$ on the $R_{5}$ calculation. Two important observations can be made. First, the early members of the manifold of calculated $\left(\pi, n \pi^{*}\right)$ states, e.g., the first five states on the column $R_{5}$, form a series with energies which can be fitted to the Rydberg formula with effective quantum numbers $n^{*}=2.907$, $3.907, \ldots, 6.907$. Next, the state at $10.21 \mathrm{eV}$ is obviously stable with respect to the addition of diffuse functions to the basis. We will see later that this state is also stable under changes in the valence part of the basis.

To characterize this $\left(\pi, \pi^{*}\right)$ state at $10.21 \mathrm{eV}$, we look at the second moment of the $\pi^{*}$ orbitals and dipole transition moments from the ground state for the states of the $R_{5}$ calculation listed in Table $I$. The second moments are shown in Table $\Pi$ and those of the $1 \pi^{*}, 2 \pi^{*}$, $\ldots, 5 \Pi^{*}$ orbitals are characteristic of successive members of a Rydberg series. The $6 \pi^{*}$ orbital has a large second moment of $35 a_{0}^{2}$. This large value for the second moment reflects considerable Rydberg character which must be present due to orthogonality requirements to the lower-energy Rydberg series $1 \pi^{*}$ to $5 \pi^{*}$. In Table III, we list the oscillator strengths for the transitions into the $\left(\pi, n \pi^{*}\right)$ states of the calculation with the $R_{5}$ basis. ${ }^{13}$ We now see the important result that the state at $10.21 \mathrm{eV}$ carries a large oscillator strength even though the large value of the second moment of the $\pi^{*}$ orbital reflects the presence of considerable Rydberg character. The large value of the transition moment, and hence the oscillator strength, indicates that the "stabilized" state at $10.21 \mathrm{eV}$ also has significant valencelike components.

The results of Table I show that the state at $10.21 \mathrm{eV}$

TABLE II. Second moment of the $\pi^{*}$ orbitals. $^{\mathrm{a}}$

\begin{tabular}{lc}
\hline \hline Orbital & $\left\langle n \pi^{*}\left|x^{2}\right| n \pi^{*}\right\rangle^{\mathrm{b}}$ \\
\hline $1 \pi^{*}$ & 42.4 \\
$2 \pi^{*}$ & 175.1 \\
$3 \pi^{*}$ & 474.8 \\
$4 \pi^{*}$ & 2081.9 \\
$5 \pi^{*}$ & 8595 \\
$6 \pi^{*}$ & 35.3 \\
$7 \pi^{*}$ & 7.1 \\
\hline \hline aror those states listed in column $R_{5}$ of \\
Table I. \\
bIn a.u. The molecule is in the $y z$ plane.
\end{tabular}


TABLE III. Oscillator strengths for the $\Psi(n \pi *) .^{\mathrm{a}}$

\begin{tabular}{cl}
\hline Transition $^{\mathrm{b}}$ & $f^{0}$ \\
\hline$\Psi \rightarrow \Psi\left(1 \pi^{*}\right)$ & 0.112 \\
$\Psi\left(2 \pi^{*}\right)$ & 0.077 \\
$\Psi\left(3 \pi^{*}\right)$ & 0.038 \\
$\Psi\left(4 \pi^{*}\right)$ & 0.003 \\
$\Psi\left(5 \pi^{*}\right)$ & 0.0001 \\
$\Psi\left(6 \pi^{*}\right)$ & 0.456 \\
$\Psi\left(7 \pi^{*}\right)$ & 0.139 \\
\hline
\end{tabular}

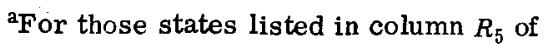
Table I. See Text.

$\mathrm{b}^{\mathrm{b}} \Psi(n \pi *)$ denotes the $(\pi, n \pi *)$ state.

$\mathcal{G}=\frac{2}{3} \Delta E\left|\left\langle 0\left|\Sigma_{i} Z_{i}\right| \Psi\left(n \pi^{*}\right)\right\rangle\right|^{2}$.

has been stabilized with respect to extensive changes in the Rydberg character of the orbital basis. To study the stability of this state under changes in the valence part of the basis, we carried out additional calculations using a $[4 s 3 p 1 d / 2 s]$ basis $^{14}$ augmented with the same five diffuse $p_{x}$ basis functions on the $\mathrm{C}$ atom as in the $R_{5}$ calculation of Table I. These calculations listed under $R_{5}^{*}$ in Table I gave five Rydberg states at almost identical energies as those of Table I and a stabilized state at 10.21 eV. The oscillator strength of the transition to this state is again 0.46. Notice also the apparent stabilized state at $\sim 21.1 \mathrm{eV}$ of the Rydberg calculations is not stable with respect to variation of the valence basis components. We, therefore, conclude the state embedded in the continuum at $10.21 \mathrm{eV}$ is stable with respect to changes in both the Rydberg and valence character of the basis, and is the only such state within $10 \mathrm{eV}$ of the Hartree-Fock continuum.

We now ask, what is this state at $10.21 \mathrm{eV}$ ? It is much more valence-like than the lower energy states and is embedded in the continuum. We believe that this is the analog to the spectroscopic $V$ state in HartreeFock theory. The excitation energy of $10.21 \mathrm{eV}$ is about $2.6 \mathrm{eV}$ larger than the observed value of $7.66 \mathrm{eV}$. The incorrect description arising in the Hartree-Fock calculation is probably due to the larger correlation effects in the ionic $V$ state than in the Rydberg states. A procedure for obtaining an accurate description of the $V$ state would be to carry out a configuration interaction on this SCF $\left(\pi, 6 \pi^{*}\right)$ state. The major effect will be the correlation between the $\Sigma-\Pi$ system and the "unmixing" of Rydberg and valence characters which would appear as extensive mixing of $\left(\pi, 6 \pi^{*}\right)$ with the lower "Rydberg" SCF states. The mixing of $\left(\pi, 6 \pi^{*}\right)$ and $\left(\pi, \pi^{*}\right)$ is so extensive, in fact, that the true $V$ state is nearly a $50-50$ mix of these SCF states. The observed $f$ value for the $N \rightarrow V$ system is 0.34 . In these calculations we can expect an increase from the SCF value of 0.46 for the oscillator strength due to the removal of Rydberg character from the states and a decrease due to the effects of electron correlation. ${ }^{5,6}$

\section{DISCUSSION AND CONCLUSIONS}

The purpose of the present work was to locate the analog to the spectroscopic $V$ state in Hartree-Fock theory and to understand its character and location in terms of a simple model. We believe that we have located such a state at $10.21 \mathrm{eV}$ which is in fact a resonance embedded in the ionization continuum with an approximate oscillator strength of 0.46 . However, the state has considerable diffuse character due to contamination with the lower-energy Rydberg states (due to orthogonality requirements). This resonance state could be a convenient starting point for a configuration interaction calculation.

The existence of such $V$ or ionic states in the HartreeFock spectrum will be a warning in studying other systems. For example, preliminary calculations on the [core] $\pi_{u}^{4} 3 \sigma_{g}^{2}-[$ core $] \pi_{u}^{3} 3 \sigma_{g}^{2} n \pi_{g}\left({ }^{1} \Sigma_{u}^{+}\right)$transitions in $N_{2}$ indicate the same behavior of the intravalence $\pi_{u}-\pi_{g}$ transition as in $\mathrm{C}_{2} \mathrm{H}_{4}$. Here again, all the $\pi_{u}^{3} n \pi_{g}\left({ }^{1} \Sigma_{u}^{+}\right)$states lying below the ${ }^{2} \Pi_{u}$ ion of $\mathrm{N}_{2}$ are members of a Rydberg series while a valence-like $\pi_{u}^{3} \pi_{s}$ state lies in the continuum. The presence of such "resonances" which are spuriously pushed into the continuum in SCF theory must be dealt with carefully in methods which use discrete spectral distributions to derive photoionization cross sections, e.g., by a Stieltjes imaging procedure. ${ }^{15}$

${ }^{1}$ R. J. Buenker and S. D. Peyerimhoff, Chem. Phys, 9, 75 (1975).

${ }^{2}$ C. F. Bender, T. H. Dunning, Jr., H. F. Scheffer III, W. A. Goddard III, and W. J. Hunt, Chem. Phys. Iett. 15, 171 (1972).

${ }^{3}$ T. H. Dunning, Jr., W. J. Hunt, and W. A. Goddard III, Chem. Phys. Lett. 4, 147 (1969).

${ }^{4} \mathrm{E}$. Miron, B. Raz, and J. Jortner, Chem. Phys. Lett. 6, 563 (1970).

${ }^{5}$ Larry E. McMurchie and Ernest R. Davidson, J. Chem. Phys. (accepted for publication).

${ }^{6} \mathrm{~T}$. Shibuya and V. McKoy, J. Chem. Phys. 54, 1738 (1971).

${ }^{7}$ K. Tanaka, Int. J. Quantum Chem. 6, 1087 (1972).

${ }^{8}$ S. Iwata and K. F. Freed, J. Chem. Phys. 61, 1500 (1974).

${ }^{9}$ Harold Basch and Vincent McKoy, J. Chem. Phys. 53, 1628 (1970).

${ }^{10}$ H. S. Taylor, Adv. Chem. Phys. 18, 91 (1970).

${ }^{11}$ E. R. Davidson and L. Z. Stenkamp, Int. J. Quantum Chem. S10, 21 (1976).

${ }^{12}$ T. H. Dunning, Jr. and P. Jeffrey Hay, Modern Theoretical Chemistry edited by H. F. Schaeffer III, (Plenum, New York, 1977) Vol. 2. The $[3 s]$ basis is the contracted segmented basis set obtained from a $(9 s 5 p)$ primitive basis.

${ }^{13}$ The ground state and excited state SCF orbitals are not orthogonal. To evaluate the transition moment, we take vectors of a particular excited state calculation. These vectors also give good approximations to the other states including the ground state. The transition moments are calculated with these eigenvectors and are sufficiently accurate for the present purposes.

${ }^{14}$ T. H. Dunning, Jr., J. Chem. Phys. 53, 2823 (1970).

${ }^{15}$ P. W. Langhoff, Int. J. Quantum Chem. S8, 347 (1974). 